\title{
The importance of movement variability for performance and prevention of injury
}

$\mathrm{R}$ ather than considering variability in a motor task as unwanted noise or a problem that must be corrected, variability in movement patterns is now seen as a functional and indeed an important part of the adaptability of coordination and movement efficiency required in producing optimal outcomes in dynamic performance. For physiotherapists, the importance of including variability in exercise techniques and programmes is becoming more and more apparent.

\section{Movement variability and movement tasks}

Movement variability can be described as the normal variations that occur in motor performance across multiple repetitions of a task (Stergiou and Decker, 2011). That is, when a person repeats a movement task, no two repetitions will be identical. Rather, each repetition will involve unique non-neural and motor patterns or, as described by Bernstein et al (1967), 'repetition without repetition'.

However, for many years, some health practitioners interested in movement have continued to focus on reducing variability, or purported 'error', during performance of movement tasks. For example, patients presenting with pain or movement dysfunction are told to 'correct' their pattern of movement and asked to perform repetition after repetition of a task-specific movement with the aim of achieving 'consistency' or a 'normal pattern'. While there are obvious advantages to asking patients to repeat movements, it could also be argued that encouraging people to focus on trying to produce the same movement patterns may simply encourage inflexible motor behaviours that subsequently limit the patient's ability to adapt to different tasks or changing environmental demands (Stergiou and Decker, 2011).

\section{Movement variability and pain/injury}

Much research has been devoted to identifying movement patterns that predispose both athletes and nonathletes to injury. However, more recently, increasing attention has been directed towards analysing patterns of movement during functional tasks instead of discrete kinematic measurements in order to gain a greater understanding of the interactions that occur in the motor system that may result in pain or injury. From this type of research, there is evidence to suggest that a loss of movement variability in fundamental movement variables leads to injury and pain (Seay et al, 2011; Hamill et al, 2012).

Hamill et al (2012) argue that absolute coordination, with its low variability, causes forces to be distributed across small surface areas, possibly resulting in overuse injuries. In contrast, the variations present during relative coordination allow joint or tissue forces to be more widely distributed, which may reduce the risk of overuse injuries. Supporting this argument is the study by Seay and colleagues (2011), which demonstrated that runners with low back pain had lower coordinative variability between their trunk and pelvis than runners without low back pain or those runners who had recovered from low back pain. There is also evidence to suggest that patients with spinal pain adopt more rigid postural control strategies (Madeleine et al, 2008) and less flexible strategies during complex tasks (Claeys et al, 2011).

Patients with anterior cruciate ligament (ACL) deficient knees have been found to have less divergence in
'When

prescribing exercises, the importance of movement variability for skilled performance and its

relationship to injury should be considered.'

flexion and extension movement during gait, indicating a more constrained type of motor behaviour (Moraiti et al, 2007). This increased behavioural rigidity may be a mechanism contributing to the eventual development of osteoarthritis in the ACL deficient knee. While there is evidence to suggest that different coordinative patterns during other sports-related activities predict injury, more prospective studies are needed to determine the role of movement variability in risk for injury.

\section{Movement variability and} skilled performance

In multi-joint tasks that require precision of an 'end-point effector', the variability of individual segment trajectories may be substantially 
greater than the variability of the endpoint trajectory (Bernstein et al, 1967; Davids et al, 2004; Horan et al, 2011). This feature is perhaps best illustrated in the classic study of Bernstein (1967), where the movement of professional blacksmiths is described when repeatedly hitting a chisel. Considerable variability was observed for individual joints of the upper body, yet the trajectory of the hammer tip was consistent with each strike, particularly at the point of impact.

Similar features have now been found during the golf swing (Horan et al, 2011) and the basketball free throw (Button et al, 2003), where different coordinative patterns exist for the more proximal segments, but at the point of impact, in the case of the golf swing for example, segmental variability is low.

Many constraints interact to shape postural behaviours during sporting endeavours, including body properties, support surfaces and tasks, but highly skilled performers are able to adapt to these constraints and perturbations, resulting in the necessary flexibility and adaptability to operate proficiently in a variety of performance development and learning contexts.

\section{Implications for health professionals}

Rather than focusing on asking patients to perform repetitive movement tasks with the goal of simply trying to rep- licate the exact same kinematics, health professionals should consider encouraging their patients to adopt flexibility in motor patterns and also take into account the patient's individual coordination strategies and recognise that these strategies are a function of the interaction between a number of constraints.

When prescribing exercises, the importance of movement variability for skilled performance and its relationship to injury should be considered. The health professional should also consider whether or not they are in fact limiting their patient's coordinative strategies by simply asking them to repeat 'correct' movement patterns during specific tasks. There is still a long way to go in understanding the complexity of movement. However, a consideration of the neurophysiological benefits of encouraging movement variability is certainly warranted. IJTR

Bernstein IH, Schurman DL, Forester G (1967) Choice reaction time as a function of stimulus uncertainty, response uncertainty, and behavioral hypotheses. Journal of Experimental Psychology 74(4): 517-24. doi: $10.1037 / \mathrm{h} 0021279$

Bernstein NA (1967) The Co-ordination and Regulation of Movements. Pergamon Press, Oxford

Button C, MacLeod M, Sanders R, Coleman S (2003) Examining movement variability in the basketball free-throw action at different skill levels. Res $Q$ Exerc Sport 74(3): 257-69

Claeys K, Brumagne S, Dankaerts W, Kiers H, Janssens L (2011) Decreased variability in postural control strategies in young people with non-specific low back pain is associated with altered proprioceptive reweighting. Eur J Appl Physiol 111(1): 115-23. doi: 10.1007/ s00421-010-1637-x

Davids K, Shuttleworth R, Button C, Renshaw I, Glazier P (2004) "Essential noise" enhancing variability of informational constraints benefits movement control: A comment on Waddington and Adams (2003). Br J Sports Med 38(5): 601-5. doi: 10.1136/ bjsm.2003.007427

Hamill J, Palmer C, Van Emmerik RE (2012) Coordinative variability and overuse injury. Sports Med Arthrosc Rehabil Ther Technol 4(1): 45. doi: 10.1186/1758-2555-4-45

Horan SA, Evans K, Kavanagh JJ (2011) Movement variability in the oolf swing of male and female skilled golfers. Med Sci Sports Exerc 43(8): 1474-83

Madeleine P, Mathiassen SE, Arendt-Nielsen L (2008) Changes in the degree of motor variability associated with experimental and chronic neck-shoulder pain during a standardised repetitive arm movement. Exp Brain Res 185(4): 689-98

Moraiti C, Stergiou N, Ristanis S, Georgoulis AD (2007) ACL deficiency affects stride-tostride variability as measured using nonlinear methodology. Knee Surg Sports Traumatol Arthrosc 15(12): 1406-13

Seay JF, Van Emmerik RE, Hamill J (2011) Low back pain status affects pelvis-trunk coordination and variability during walking and running. Clin Biomech (Bristol, Avon) 26(6): 572-8. doi: 10.1016/j.clinbiomech.2010.11.012

Stergiou N, Decker LM (2011) Human movement variability, nonlinear dynamics, and pathology: Is there a connection? Hum Mov Sci 30(5): 869-88. doi: $10.1016 / \mathrm{j}$. humov.2011.06.002

Kerrie Evans, Senior lecturer, School of Allied Health Sciences, Griffith University, Gold Coast Campus and Menzies Health Institute Queensland, Australia;

Neil Tuttle, Senior lecturer, School of Allied Health Sciences, Griffith University, Gold Coast Campus and Menzies Health Institute Queensland, Australia.

\section{Clinical Supervision for Pallative Care}

Clinical supervision has been researched, promoted, and proven as a very effective strategy for constantly developing skills, for maintaining and raising standards, for encouraging personal and professional development, and for building team ethos. This book explores clinical supervision and the qualities, skills, models and ethics needed to ensure success.

Clinical Supervision for Palliative Care has been written as a workbook. You will find pauses for reflection and lots of questions to consider and answer. Good Palliative Care is based on good communication - so you are asked to interact with the text.

Researched with a range of professionals working in the field, and with some of those receiving their care, this book encourages implementation of Clinical Supervision across the widening field of Palliative Care - not only because it will support and sustain practitioners, who are not always good at looking after themselves, but because it will also fulfil the ultimate goal of all of us - the best possible care of dying people, helping them to the death that they want, and easing their suffering and that of those closest to them.

ISBN-13: 978-1-85642-291-8; 234 x 156 mm; paperback; 144 pages; publication 2006; £24.99

Order your copies by visiting www.quaybooks.co.uk or call our Hotline $+44(0) 1722716935$ 
Copyright of International Journal of Therapy \& Rehabilitation is the property of Mark Allen Publishing Ltd and its content may not be copied or emailed to multiple sites or posted to a listserv without the copyright holder's express written permission. However, users may print, download, or email articles for individual use. 